\title{
Can Grade-6 students understand quarks? Probing acceptance of the subatomic structure of matter with 12-year-olds
}

\author{
Gerfried J. Wiener ${ }^{1,2}$, Sascha M. Schmeling ${ }^{1}$ and Martin Hopf ${ }^{2}$ \\ ${ }^{1}$ CERN, European Organization for Nuclear Research, Geneva, Switzerland \\ ${ }^{2}$ University of Vienna, Austrian Educational Competence Centre Physics \\ For correspondence: gerfried.wiener@cern.ch
}

\begin{abstract}
This study introduces a teaching concept based on the Standard Model of particle physics. It comprises two consecutive chapters - elementary particles and fundamental interactions. The rationale of this concept is that the fundamental principles of particle physics can run as the golden thread through the whole physics curriculum. The design process was conducted from a constructivist perspective based on students' documented conceptions. Three pillars underpin the whole teaching concept: a permanent model character, linguistic accuracy, and innovative typographic illustrations. Using the framework of design-based research, microteaching sessions with 20 Grade- 6 students were conducted to probe its acceptance. The study focusses on learning processes of 12-year-olds with respect to elementary particles. Our findings indicate broad acceptance of most key ideas, but also avoidance when considering the permanent model character of physics. The most promising outcomes of the study are pure typographic illustrations. Not only were these thoroughly accepted by all students, but they also seem to reduce known misconceptions. Overall, students' understanding of elementary particles improved fundamentally.
\end{abstract}

Keywords: particle physics, elementary particles, fundamental interactions, acceptance, teaching concept

\section{Introduction}

According to the "Trends in International Mathematics and Science Study" (TIMSS, Mullis et al., 2012), in most countries the chapter of modern physics is placed at the end of curricula, if at all. Since these chapters - particularly the Standard Model, the "first full deck of cards to play with" (Griffiths, 2004: 3) - describe processes fundamental to physics, this situation might not support high school students' development of a coherent knowledge structure. We therefore propose a new teaching concept that reflects particle physics' position as the basis of all other physics. The proposed concept comprises two short chapters as a starting point for physics teaching. The first chapter tackles the particulate nature of matter by focusing on a state-of-the-art atomic model. This leads to a brief outline from electrons down to quarks. The second chapter introduces the fundamental interactions and their associated bosons. Since it is argued that thinking in and with models is an essential component of an appropriate science knowledge (Mikelskis-Seifert \& Fischler, 2003a; Gilbert, 2004), the aim of the proposed concept is to focus on the permanent model character of physics. We consider both chapters to be prototypical for such a model-based approach to physics teaching.

Key to the concept's design is its independence from today's curricula. Given that every physics phenomenon can be traced back to fundamental interactions between elementary particles, the integration of the proposed concept could be adequate for any age group. Ideally, one could use this concept from the very beginning of physics teaching by introducing particle physics to 12-year-olds. This allows the fundamental principles of particle physics to run as the golden thread through the whole physics curriculum.

The design process of the teaching concept was approached from a constructivist viewpoint: students are enabled to construct knowledge on their own on the basis of the material provided. This process is 
based on students' pre-existing cognitive structures. The exploration and consideration of students' conceptions is essential to avoid triggering misconceptions. At the same time, this knowledge provides a base for the development of adequate learning opportunities. Thus, an understandable and appropriate offer can be made to the students, enabling them to construct consistent knowledge (Duit, 1996; Duit \& Treagust, 2003).

State of research

Studies of students' conceptions about the particulate nature of matter originated in the 1980s, mainly in chemistry research regarding molecules and atoms (Pfundt, 1981; Novick \& Nussbaum, 1981; Renstroem, 1987; Andersson, 1990; Stavy, 1991; de Vos \& Verdonk, 1996; Harrison \& Treagust, 1996; Harrison \& Treagust, 2000; Nakhleh \& Samarapungavan, 1999). These first studies showed that only few students use a particle model to explain physics phenomena. Especially when dealing with everyday phenomena, a particle model is neglected (Pfundt, 1981; Novick \& Nussbaum, 1981; Stavy, 1991). However, when offered as a possible explanation, students broadly accept a particle model (Harrison \& Treagust, 2000; Snir et al., 2003). Equally striking is a significant age dependence when it comes to the acceptance of a particle model (Dow et al., 1978; Pfundt, 1981; Harrison \& Treagust, 1996). In contrast, a variety of age-independent misconceptions of the atomic structure of matter have been documented (Novick \& Nussbaum, 1981; Boz, 2006).

Everyday experience favours a continuous perception of the world. Therefore, most students' conceptions are dominated by a continuum perspective. When introduced to a particle model, most students try to combine both models. This leads to a frequent mixing and overlapping of continuum and discontinuum conceptions, whereby students try to integrate the novel particle model into the framework of the existing continuum model (Pfundt, 1981; Renstroem, 1987; Andersson, 1990; Boz, 2006). These misconceptions can be supported by erroneous illustrations in textbooks. For example, Andersson (1990) describes their impact by discussing an illustration of a glass of water filled with $\mathrm{H}_{2} \mathrm{O}$ molecules floating around in the water.

Further studies also show that despite students' acceptance of a particle model, two misconceptions prevail: no reproduction of the permanent motion of particles, and the negation of the existence of empty space (Novick \& Nussbaum, 1981; Renstroem, 1987; Andersson, 1990; Harrison \& Treagust, 1996). Furthermore, numerous studies have shown that teaching a particle model leads to an automatic transfer of macroscopic aspects and daily life experiences into the world of particles. Students think of particles with faces, while water molecules are thought of as wet and blue (Renstroem, 1987; Andersson, 1990; Boz, 2006; Ozmen, 2011).

\section{Concept design}

The concept's design is based on the above documented studies on students' conceptions. Griffith \& Preston (1992) gave an overview of 52 different misconceptions related to the properties of atoms and molecules, most of which are supported by erroneous figures and illustrations. To avoid triggering any of these misconceptions and to ensure comprehensiveness and coherence, the teaching concept is based on three pillars:

\section{Permanent model character}

A big challenge of teaching particle physics is its abstractness. Therefore, demonstration experiments are limited, the precision of explanations hard to balance with their adequateness, and, due to the inconceivable size ratio, realistic illustrations are doomed to fail. But this abstractness enables one feature to stand out, namely, the permanent model character of science originally defined by Hertz (1899): "When from our accumulated previous experience we have once succeeded in deducing images of the desired nature, we can then in a short time develop by means of them, as by means of models, the consequences which in the external world only arise in a comparatively long time, or as the result of our own interposition." Ever since, modelling has been considered to be a key process in the development of scientific knowledge (Ornek, 2008; Chittleborough \& Treagust, 2009; Justi, 2009). That is why we have chosen to focus on the permanent model character of particle physics. 
Constantly pointing out that everything just said describes a model can enable a proper comprehension of physics.

Consequently, this should lead to setting a tone for the fundamental nature of science and laying foundations for all topics to follow. The phrase "With this model, we describe..." plays a big role in the teaching concept and is frequently repeated and emphasised.

\section{Linguistic accuracy}

Another difficulty in the field of modern physics arises when speaking about it. First of all, one has to jump back and forth between a language of science and our everyday language (Rincke, 2010). This requires careful definitions of certain wordings to maintain their original meaning, a process often neglected in classrooms. Secondly, particle physics is still quite a young field of physics. Many of today's wordings and phrasings originate from the early days of particle physics about 100 years ago. For example, any description of "circular orbits" within the Bohr model can be considered to be anachronistic and therefore act as a possible source of misconceptions (Karsten et al., 2011). The same problem applies to the historical accumulation of hundreds of "elementary particles" in the so-called particle zoo. Therefore, clear-cut language is needed to offer valuable teaching material.

Accordingly, linguistic accuracy is a prominent aspect of our concept. This was largely addressed by minor changes to specific phrasings. For instance, when introducing the atomic model, instead of "the nucleus" we refer to "the nucleus-space". Doing so supports the location aspect of the nucleus-space, while neglecting its manifested aspects. The same idea is applied when the orbital-space is introduced. These areas are then characterised by the phrase "In this space it is possible to locate certain particles". Thus, these changes aim to increase the probability aspect of particles.

When talking about particles, the proposed concept distinguishes between particles and particle systems. This means that only elementary particles, such as leptons and quarks, are denoted as particles. In contrast, hadrons count as particle systems which are made of particles. However, particle systems can still be described as particle-like objects with particle-like properties.

\section{Pure typographic illustrations}

In addition to linguistic accuracy, the concept relies on carefully constructed illustrations. Educational research shows that visual representations are essential for communicating ideas in the science classroom (Carney \& Levin, 2002; Cook, 2006). However, as mentioned above, realistic illustrations in the field of particle physics are, by definition, doomed to fail. Two major difficulties prevail: the sheer scale of atoms, and any graphic illustrations of particles and atoms. Using interactive animations (Huang \& Huang, 2012) and animated movies (Eames \& Eames, 1977) can help to overcome the problem of demonstrating the inconceivable scale of atoms. As for static illustrations, there is no helpful solution in sight.

For the general illustrations of particles and particle systems we propose a typographic approach. Having the permanent model character in mind all particles and particle systems are thus represented by their respective symbol (figure 1). 

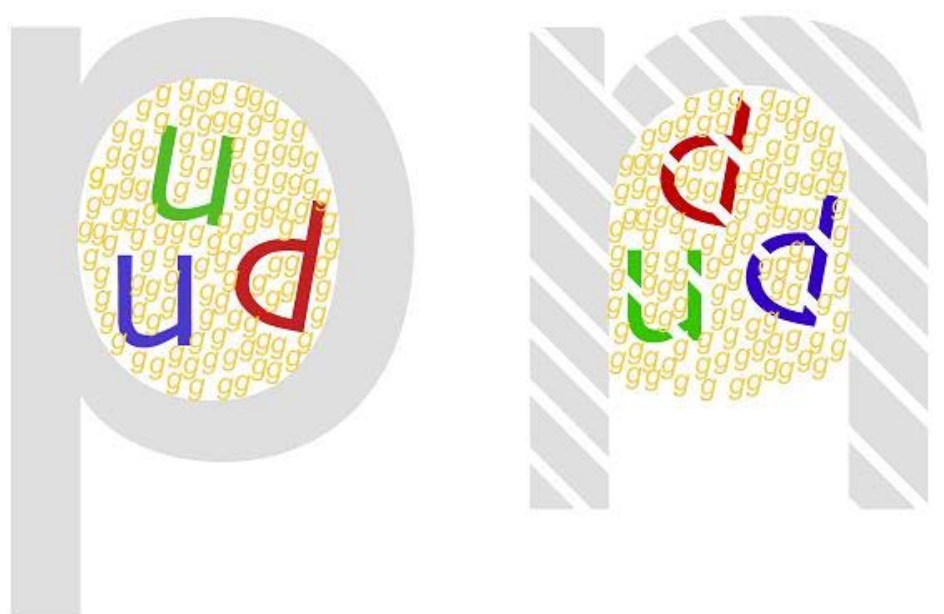

Figure 1. Typographic illustrations of a proton and an antineutron.

The goal of this approach is to avoid misconceptions of three-dimensional particles, while referring to symbols as defined objects. Furthermore, the underlying colour scheme of the proposed concept is intended to distinguish particles from particle systems. Quarks are blue, green, and red. In the first chapter, this serves the sole purpose to identify quarks as elementary particles. This sets up the notion of colour charge to be introduced in the second chapter. Since antiparticles are also part of the concept, the need for a visualisation of anticolour charge arises. The proposed implementation avoids the commonly-used complementary colours. Instead, antiparticles and antiparticle systems are identified through stripes. This strategy provides a simple distinction while bypassing the use of misleading complementary colours. For instance, white-striped green is used instead of magenta as a more adequate depiction of anti-green.

A typographic approach is also used to illustrate the atomic model. Both the nucleus-space and the orbital-space are displayed using their names, the latter being made to look spherical. A shift of focus is created by using the two descriptive words themselves to illustrate the different spaces. This gives the impression of a three-dimensional atomic model while reducing the possible misimpression of orbits or shells (figure 2).

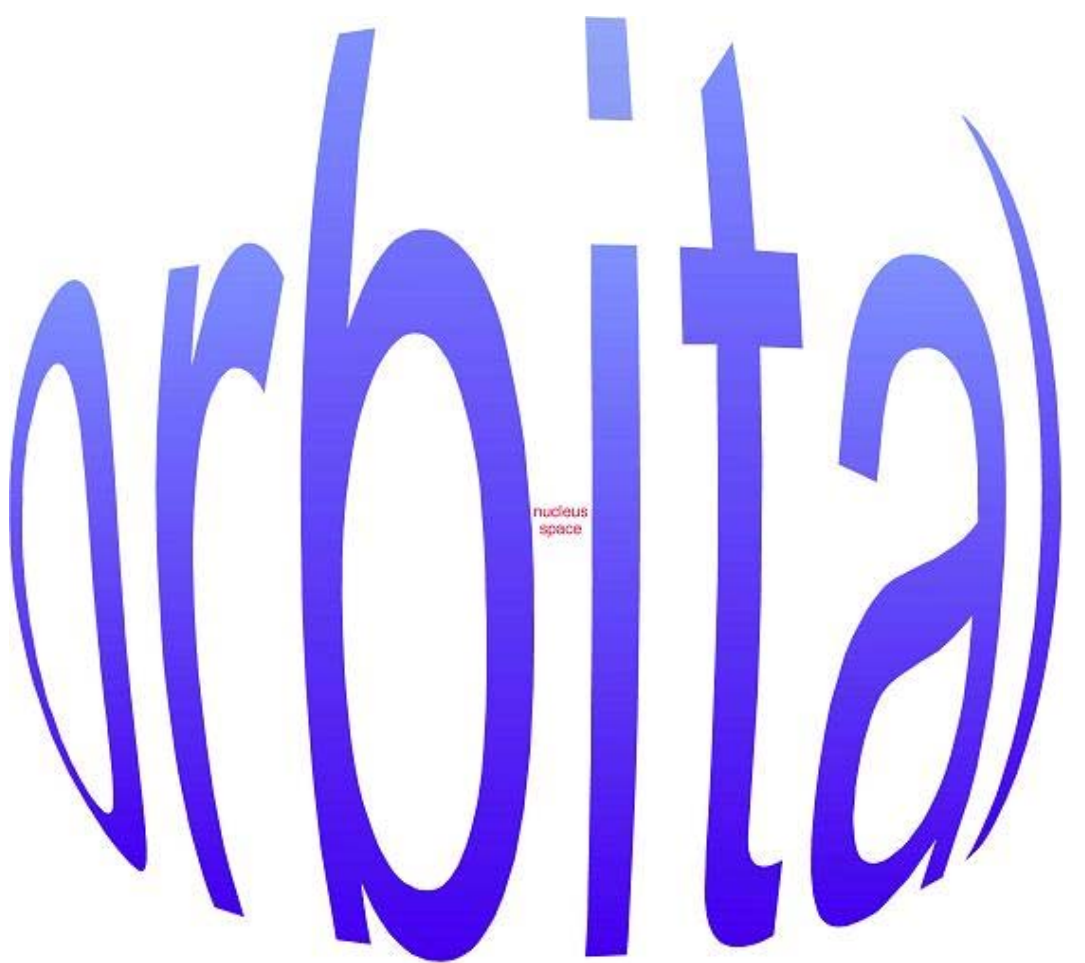

Figure 2. Typographic illustration of an atomic model. 
Admittedly, this illustration has its limitations. First, the visualisation focuses solely on the quantitative aspects of the atomic model, its aim being to illustrate the distinction between the nucleus-space and the orbital-space. Second, it does not overcome the problem of a realistic size ratio, and it requires a careful introduction by the teacher to explain the underlying model character. Also, it does not convey the notion of different orbital shapes within the specific orbital-space, which must be introduced at a later stage in the physics curriculum.

\section{Methods}

A study was performed to evaluate the effects of the teaching concept by probing its acceptance with 12-year-olds. Having only had very little physics education, such students can be considered as novices, especially with respect to particle physics. The main purpose of these teaching experiments was to evaluate students' general understanding of the concept, and to find out whether students use it for problem-solving.

\section{Probing acceptance}

The technique of probing acceptance (Jung, 1992) was developed to investigate learning processes. It comprises a set of microteaching sessions, including planned interview phases. Explanations are offered, then observations made regarding students' understanding, valuation, and use of this information in solving problems. The aim is to identify resistances to elements of the instruction. An advantage of this setting compared to conventional problem-centered interviews is the reduction of short-term, ad-hoc constructs (Wiesner \& Wodzinski, 1996).

Each microteaching session consists of four steps. First, specific information about the concept being addressed is offered to the student in the form of, for example, an experiment, presentation or short lecture. The student then evaluates the plausibility and comprehensibility of the information offer. This is followed by the task of paraphrasing the presented information "in own words". The student's final task is to apply the new knowledge to concrete examples. Based on the student's reactions, one can then identify obstructive aspects of the original explanations given. Using the framework of design-based research, perpetual redesigning and retesting eventually leads to understandable and valuable teaching material (Design-Based Research Collective, 2003).

\section{Study design, setting and analysis}

The method of this study relies on the technique of probing acceptance. We focused on the first chapter of the teaching concept, which introduces the subatomic structure of matter. Based on the atomic model's introduction, several key ideas were formulated. A first test survey was then conducted with four Austrian Grade- 6 students to gain insight into the feasibility of the study. As a result, the information offer was slightly revised and modified. This was followed by another set of microteaching sessions with eight Grade- 6 students. After evaluation of the transcripts, an extensive redesign process was required. The frequency of references to the permanent model character in the information offer was increased, the typographic illustrations were redesigned more precisely, and the total number of key ideas was reduced to a more feasible amount. The final information input covers these eleven key ideas:

I. Matter is everything that can be touched, practically or theoretically.

II. Reality is described through models.

III. There are atoms (Democritus - átomos).

IV. Atoms are divided into two areas: the nucleus-space and the orbital-space.

V. In the nucleus-space there are protons and neutrons.

VI. Protons and neutrons are particle systems made of quarks.

VII. Quarks are indivisible. In this model, these are called elementary particles.

VIII. In the orbital-space electrons can be found.

IX. Electrons are indivisible. In this model, these are called elementary particles.

X. Apart from particles, there is only empty space.

XI. There are (different) atoms, which may combine to form compounds. 
The main study took place in spring 2014 at an Austrian high school with another eight Grade-6 students. Each microteaching session lasted about 40 minutes (table 1) and was videotaped using a GoPro® camera. At the beginning, the information was offered through a ten-minute talk. Key descriptive ideas, such as the distinction between nucleus-space and orbital-space, were supported by accompanying typographic illustrations. This presentation was followed by the student's task of evaluating and paraphrasing the information offer, which usually took about five minutes. Of main interest were the intelligibility of the key ideas, and the feasibility of the teaching concept.

Table 1. Timeframe for the microteaching sessions.

\begin{tabular}{llr}
\hline$\#$ & Phase & Duration \\
\hline 1 & Information input & $10^{\prime}$ \\
2 & Evaluation \& Paraphrasing & $5^{\prime}$ \\
3 & Transfer example 1 \& Evaluation & $12^{\prime}$ \\
4 & Transfer example 2 \& Evaluation & $12^{\prime}$ \\
\hline
\end{tabular}

Next, two concrete examples were given. At first, some grains of salt were sprinkled across the table. The task was to explain whether salt can be identified as matter, and what it is made of. The second example asked the same question but used droplets of water instead of salt grains. Thus, the student's insight into two different states of matter could be evaluated.

The method of qualitative content analysis (Mayring, 2010) was applied to evaluate the findings. Category-based analysis was carried out on all interviews. Three categories (fully $[\checkmark]$; partially $[\sim]$; not adequate $[x]$ ) were used, with the addition of a fourth non-mention ([ ]) category due to a few omissions. The intercoder-reliability resulted in $\mathrm{K}=0.725$, meeting the required standard.

\section{Results}

The final version of the information offer led to quite successful results. The concept's chapter on the atomic model was broadly accepted by all students. Most key ideas were used for problem-solving and most of them were rated to be plausible. We present here the most important and, from a teaching perspective, the most interesting findings.

Key idea I, the crucial introduction of matter as the main subject of the concept was very successful and showed broad acceptance from the beginning (table 2). By linking it to the haptic action of touching, a general understanding of matter was enabled. Doing so allowed for even more difficult questions concerning different materials to be answered correctly. For example, in response to the question whether air qualifies as matter, many students agreed by referring to wind as moving air touching their face. When asked to name the counterpart of matter most students concluded that everything is matter. Additionally, some students even mentioned the absence of matter in outer space, for example as follows: "If one flies with a rocket into outer space, there is nothing. I believe, there is no matter anymore. Except for stars of course." (All interview quotes have been translated from the original German version.)

Table 2. Overview of the students' evaluation of key idea I: Matter is everything that can be touched, practically or theoretically.

\begin{tabular}{lcccccccc}
\hline Phase & S01 & S02 & S03 & S04 & S05 & S06 & S07 & S08 \\
\hline Paraphrasing of the key idea & $\checkmark$ & $\checkmark$ & $\checkmark$ & $\sim$ & $\checkmark$ & $\checkmark$ & $\checkmark$ & $\checkmark$ \\
Transfer of the key idea (salt) & $\checkmark$ & $\checkmark$ & $\checkmark$ & $\checkmark$ & $\checkmark$ & $\checkmark$ & $\checkmark$ & $\checkmark$ \\
Transfer of the key idea (water) & $\checkmark$ & $\checkmark$ & $\checkmark$ & $\checkmark$ & $\checkmark$ & $\checkmark$ & $\checkmark$ & $\checkmark$ \\
\hline
\end{tabular}

More complex key ideas, such as VII \& IX introducing elementary particles, were also accepted to a great extent. Since these two key ideas showed poor acceptance in the test studies, this can be traced back to the revised typographic illustrations. However, the students' acceptance and evaluation differ. While accepting their indivisibility, most students value elementary particles to be difficult to understand, as one student stated: "Somehow, I always thought that one can divide everything forever." 
This goes hand in hand with a healthy scepticism regarding their elementary state, as put so elegantly by another student: "I can imagine indivisible particles, but I think if our techniques improve maybe we can find something smaller."

Additionally, key idea $\mathrm{X}$ tackling the de facto emptiness of matter, proved to be problematic. Surprisingly, no student seemed to have any problems when talking about empty space itself. Even when describing the atomic model, the empty space was accepted repeatedly. But once it came to everyday objects, most students had to step back. One student mentioned doubts as follows: "This is really hard to imagine, because for example, the table isn't empty, there are no holes or something else in between."

Out of the eleven key ideas, only two showed poor acceptance: key idea XI focusing on compounds, such as molecules, and key idea II, which concentrates on the permanent model character. We identified different reasons for their evaluation. As for key idea II the concept's aim to emphasise the permanent model character of physics was not successful. Not only did we encounter poor acceptance, there were also many omissions. During the different stages of the sessions, most students didn't mention the model character at all (table 3). Only when specifically asked about this key idea did some of them evaluate it in an adequate way. At first, it was largely accepted, as one student stated: "You have to illustrate it somehow." But as the session progressed, more and more doubts were raised by the students, for example: "But what does it [the elementary particle] look like?" In this particular situation, the typographic illustrations proved to be helpful in overcoming the need for a realistic drawing. Overall, though, the question of how to make proper use of the permanent model character of particle physics remains.

Table 3. Overview of the students' evaluation of key idea II: Reality is described through models.

\begin{tabular}{lcccccccc}
\hline Phase & S01 & S02 & S03 & S04 & S05 & S06 & S07 & S08 \\
\hline Paraphrasing of the key idea & & $\sim$ & $\checkmark$ & $\checkmark$ & x & $\checkmark$ & $\checkmark$ & $\checkmark$ \\
Transfer of the key idea (salt) & $\checkmark$ & & $\sim$ & $\checkmark$ & & $\checkmark$ & $\sim$ & \\
Transfer of the key idea (water) & $\checkmark$ & $\sim$ & & & & $\checkmark$ & $\checkmark$ & $\checkmark$ \\
\hline
\end{tabular}

The students' evaluation of key idea XI revealed difficulties in terms of the linguistic accuracy. Most of the new words, such as quarks, orbital-space, and nucleus-space, caused no problems. But at the end of many sessions students mentioned a literary overload, for example: "Well, there are certainly some terms, for example, mole-, molec-, molep-, molecule, yes, molecule, which are definitely difficult to memorise. These special terms are really complicated." However, only a few salient transformations of elements of the explanation were observed. Most key words were used consistently as originally introduced. The only exception was the use of nucleus-space and orbital-space. Here the nucleusspace proved to appeal a lot more to students than the orbital-space. All students referred to the nucleus-space and made use of it in all repetitions. In contrast, the orbital-space was almost always transformed into "the orbital". But when asked specifically about "the orbital" the transformation vanished and the orbital-space was used again. We therefore believe it is safe to say this transformation occurs for practical reasons, but it highlights the uphill nature of the battle for linguistic accuracy.

\section{Discussion}

The explicit question motivating this work was whether Grade-6 students can understand and make use of the proposed subatomic particle concept. Indeed, we might claim that the results presented above can be judged as satisfactory. The typographic illustrations especially had a promising impact on students' learning processes. However, there are specific details that need to be addressed.

First, our aim of conveying a permanent model character was not fulfilled. Although the information input was carefully constructed from a model-based point of view, it showed little success. Only a few students adopted the proposed model-based perspective. This should come as no surprise, as 
other findings suggest that students need more experience using models as intellectual tools (Grosslight et al., 1991). Even with acceptance, many paraphrases exposed only a naive epistemological model character. Mostly, the students' interpretations focused on a model as a physical copy of reality. Thus, the descriptive model was accepted but never seriously questioned. One improvement could be to start with a specific course about models (Mikelskis-Seifert \& Fischler, 2003b), potentially leading to a more stable base upon which to build more complex chapters, such as fundamental interactions. However, for our research, the question remains as to whether the particle physics concept sufficiently supports a model-based approach of teaching physics.

Second, the linguistic modifications within the teaching concept seemed to appeal to all students. The clear distinction between particles and particle systems resulted in very positive evaluations and led to broad acceptance. In particular, the notion of protons and neutrons as particle systems made of particles recurred consistently. Nevertheless, our study has limitations in terms of linguistic accuracy. The timeframe of 40 minutes did not allow for a proper discussion of all the modified phrasings during the planned interview phases. Indeed, the literary overload noted at the end of most sessions demonstrates the need for future linguistic modifications of the teaching concept, especially given the latter's reliance on the introduction of novel terms and phrasings.

Third, the typographic illustrations proved to be comprehensible and adequate. All students evaluated them to be understandable and showed broad acceptance. Especially when it came to the distinction between particles and particle systems, the illustrations turned out to be very helpful. The underlying colour pattern, supported by the careful use of wording led to a clear distinctness. We observed no confusion regarding electrons and quarks during any session. No students showed any difficulty when talking about elementary particles, except with respect to their indivisibility. A more astonishing side effect was the observed impact on known misconceptions. Regarding the topics of empty space or the scale of atoms, we still found persistent misconceptions in accordance with documented research (Novick \& Nussbaum, 1981; Renstroem, 1987; Andersson, 1990; Harrison \& Treagust, 1996). However, over the course of two test studies and the main study, everyday descriptions of particles almost vanished. In the end, we hardly encountered any transfer of macroscopic aspects onto the properties of subatomic objects. The students' evaluations suggest this is mostly due to the revised typographic illustrations. Upcoming challenges include how to improve and implement these when introducing fundamental interactions. For future directions, we suggest typographic illustrations as the most promising outcome of this study.

Last, we want to give a brief outline of how the particle physics concept relates to previous research and how it can be used in classrooms. Its aim is to introduce elementary particles and fundamental interactions by linking them through their respective charges. This enables an early introduction of key terms such as quark, electron, charge, and interaction. One can then build the whole physics curriculum upon these fundamental principles. For obvious reasons, not all physics chapters can be explained extensively enough through particle physics. But the vast majority is adequately suited. Particularly when looking at central topics of the curriculum and how to link them to particle physics, one already finds plenty of intriguing proposals. These include, for instance, a photon-based approach of teaching optics (Gjurchinovski, 2013), an introduction of electromagnetism by using particle accelerators (Sinflorio et al., 2006), Newton's laws of motion explained through quantum physics (Ogborn \& Taylor, 2005; Pinto 2007), a course on quantum physics supported by GeoGebra simulations (Malgieri et al., 2014), and, of course, a couple of CERN-based explanations of particle physics (Long, 2011; Cid-Vidal \& Cid, 2011; Johansson, 2013; Johansson \& Watkins, 2013). This list is not exhaustive, but it serves to give a general impression. Ideally, our concept supports and enables future work to facilitate integrating particle physics into modern physics curricula.

\section{Conclusion}

In this paper, we have presented a new teaching concept based on particle physics. It comprises two consecutive chapters - elementary particles and fundamental interactions. The main motivation originates from the idea of particle physics linking the whole physics curriculum as the fundamental basis of physics. The concept's aim is to introduce modern physics at an earlier stage than is currently 
the case in most countries. The design process was conducted from a constructivist perspective based on documented students' conceptions. Three main pillars - a permanent model character, linguistic accuracy, and innovative typographic illustrations - support the whole teaching concept. To evaluate students' general understanding of the concept, a study was conducted. Based on the concept's first chapter on elementary particles, eleven key ideas were formulated. The acceptance of these key ideas was then investigated over the course of two test and one main study with 20 Grade- 6 students. Our findings were quite successful with two main outcomes of the study. On the one hand, the permanent model character was poorly accepted and barely used for problem-solving. Here, the concept needs to be adapted for future studies. On the other hand, the typographic illustrations led to broad acceptance and, in combination with linguistic accuracy, to a reduction of known misconceptions. Signs of everyday descriptions of particles largely vanished during the repetitions. We consider this to be the most interesting result of the presented study and the most promising application for teaching particle physics.

Future research will concentrate on the teachers' opinions concerning the teaching concept. A followup study is being designed to again probe acceptance of the concept with Grade- 6 students. This time, instead of education researchers, the microteaching sessions will be led by experienced teachers to evaluate their implementation of the concept. Combined with the students' acceptance, the teachers' evaluation should provide detailed information on the didactical feasibility of the teaching concept.

\section{Acknowledgements}

We thank Prof. Michael Kobel for useful discussions and Prof. Rodney Jory for valuable suggestions.

\section{References}

Andersson, B. (1990). Pupils' Conceptions of Matter and its Transformations (age 12-16). Studies in Science Education, 18, 53-85

Boz, Y. (2006). Turkish Pupils' Conceptions of the Particulate Nature of Matter. Journal of Science Education and Technology, 15, 203-213

Carney, R. N. \& Levin, J. R. (2002). Pictorial Illustrations Still Improve Students' Learning From Text. Educational Psychology Review, 14, 5-26

Chittleborough, G. D. \& Treagust, D. F. (2009). Why Models are Advantageous to Learning Science. educación química, 12-17

Cid-Vidal, X. \& Cid, R. (2011). How to count 300 trillion protons travelling at the speed of light. Physics Education, 46(3), 309-311

Cook, M. P. (2006). Visual Representations in Science Education: The Influence of Prior Knowledge and Cognitive Load Theory on Instructional Design Principles. Science Education, 90, 1073-1091

Design-Based Research Collective (2003). Design-based Research: an emerging paradigm for educational inquiry. Educational Researcher, 32, 5-8

de Vos, W. \& Verdonk, A. H. (1996). The particulate nature of matter in science education and in science. Journal of Research in Science Teaching, 33, 657-664

Dow, W., Auld, J. \& Wilson, D. (1978). Pupils' Concepts of Gases, Liquids and Solids. Dundee College of Education

Duit, R. (1996). The constructivist view in science education - what it has to offer and what should not be expected from it. Investigações em Ensino de Ciências, 1, 40-75

Duit, R. \& Treagust, D. F. (2003). Conceptual change: A powerful framework for improving science teaching and learning. International Journal of Science Education, 25(6), 671-688

Eames, C. \& Eames, R. (1977). Powers of Ten. [Online], Available: www.youtube.com/watch?v=0fKBhvDjuy0 [24.3.2015]

Gilbert, J. (2004). Models and modelling: routes to more authentic science education. International Journal of Science and Mathematics Education, 2, 115-130

Gjurchinovski, A. (2013). Reflection from a moving mirror - a simple derivation using the photon model of light. European Journal of Physics, 34, 1-4

Griffith, A. \& Preston, K. (1992). Grade-12 Students' Misconceptions Relating to Fundamental Characteristics of Atoms and Molecules. Journal of Research in Science Teaching, 29, 611-628

Griffiths, D. (2004). Introduction to elementary particles. Weinheim: Wiley-VCH

Grosslight, L., Unger, C., Jay, E., \& Smith, C. (1991). Understanding models and their use in science: Conceptions of middle and high school students and experts. Journal of Research in Science Teaching, 28, 799-822.

Harrison, A. G. \& Treagust, D. F. (1996). Secondary students' mental models of atoms and molecules: Implications for teaching chemistry. Science Education, 80, 509-534

Harrison, A. G. \& Treagust, D. F. (2000). Learning about atoms, molecules, and chemical bonds: A case study of multiple-model use in grade 11 chemistry. Science Education, 84, 352-381

Hertz, H. (1899). The principles of mechanics. London: Macmillan and Co. Ltd

Huang, C. \& Huang, M. (2012). The scale of the universe 2. [Online], Available: htwins.net/scale2 [24.3.2015]

Johansson, K. E. (2013). Exploring quarks, gluons and the Higgs boson. Physics Education, 48(1), 96-104

Johansson, K. E. \& Watkins, P. M. (2013). Exploring the standard model of particles. Physics Education, 48(1), 105-114

Jung, W. (1992). Probing acceptance, a technique for investigating learning difficulties. In R. Duit, F. Goldberg \& H. Niedderer (Ed.), Research in physics learning: Theoretical issues and empirical studies. Kiel: IPN, 278-295 
Justi, R. (2009). Learning how to model in science classroom: key teacher's role in supporting the development of students' modelling skills. educación química, 32-40

Karsten, F., Koch, T., Kranzinger, F. \& Theis, M. (2011). Planeten, Wolken oder schwarze Kisten? Wie können wir Atome in der Schule didaktisch sinnvoll beschreiben? Physik Journal, 10, 11, 39-42

Long, L. (2011). More 'hands-on' particle physics: Learning with ATLAS at CERN. Physics Education, 46(3), 270-280

Malgieri, M., Onorato, P. \& De Ambrosis, A. (2014). Teaching quantum physics by the sum over paths approach and GeoGebra simulations. European Journal of Physics, 35, 1-21

Mayring, P. (2010). Qualitative Inhaltsanalyse. Grundlagen und Techniken. Weinheim: Beltz

Mikelskis-Seifert, S. \& Fischler, H. (2003a). Die Bedeutung des Denkens in Modellen bei der Entwicklung von Teilchenvorstellungen - Stand der Forschung und Entwurf einer Unterrichtskonzeption. Zeitschrift für Didaktik der Naturwissenschaften, 9, 75-88

Mikelskis-Seifert, S. \& Fischler, H. (2003b). Die Bedeutung des Denkens in Modellen bei der Entwicklung von Teilchenvorstellungen - Empirische Untersuchung zur Wirksamkeit der Unterrichtskonzeption. Zeitschrift für Didaktik der Naturwissenschaften, 9, 89-103

Mullis, I., Martin, M., Minnich, C., Stanco, G., Arora, A., Centurino, V. \& Castle, C. (2012). TIMSS 2011 Encyclopedia: Education Policy and Curriculum in Mathematics and Science, Volumes 1 and 2. Chestnut Hill, MA: TIMSS \& PIRLS International Study Center, Boston College.

Nakhleh, M. B. \& Samarapungavan, A. (1999). Elementary school children's beliefs about matter. Journal of Research in Science Teaching, 36, 777-805

Novick, S. \& Nussbaum, J. (1981). Pupils' Understanding of the Particulate Nature of Matter: A Cross-Age Study. Science Education, 65, 187-19

Ogborn, J. \& Taylor, E. F. (2005). Quantum physics explains Newton's laws of motion. Physics Education, 40, 26-34

Ornek, F. (2008). Models in Science Education: Applications of Models in Learning and Teaching Science. International Journal of Environmental \& Science Education, 3(2), 35 - 45

Ozmen, H. (2011). Turkish primary students' conceptions about the particulate nature of matter. International Journal of Environmental \& Science Education, 6, 99-121

Pfundt, H. (1981). Das Atom - Letztes Teilungsstück oder erster Aufbaustein? Zu den Vorstellungen, die sich Schüler vom Aufbau der Stoffe machen. chimica didactica, 7, 75-94

Pinto, M. B. (2007). Introducing the notion of bare and effective mass via Newton's second law of motion. European Journal of Physics, 28, 171-181

Renstroem, L. (1987). Pupils Conceptions of Matter. A phenomenographic approach. Proceedings of the 2. Int. Seminar "Misconceptions and Educational Strategies in Science and Mathematics", 3, 400-414

Rincke, K. (2010). Alltagssprache, Fachsprache und ihre besonderen Bedeutungen für das Lernen. Zeitschrift für Didaktik der Naturwissenschaften, 16, 235-260

Sinflorio, D. A., Fonseca, P., Coelho, L. F. S. \& Santos, A. C. F. (2006). Teaching electromagnetism to high-school students using particle accelerators. Physics Education, 41(6), 539-541

Snir, J., Smith, C. L. \& Raz, G. (2003). Linking phenomena with competing underlying models: A software tool for introducing students to the particulate model of matter. Science Education, 87, 794-830

Stavy, R. (1991). Children's Ideas About Matter. School Science and Mathematics, 91(6), 240-244

Wiesner, H. \& Wodzinski, R. (1996). Akzeptanzbefragungen als Methode zur Untersuchung von Lernschwierigkeiten und Lernverläufen. Lernen in den Naturwissenschaften, 250-274 